\title{
SENP-1 enhances hypoxia-induced proliferation of rat pulmonary artery smooth muscle cells by regulating hypoxia-inducible factor- $1 \alpha$
}

\author{
FANG ZHOU ${ }^{1,2}$, AIGUO DAI $^{1}$, YONGLIANG JIANG ${ }^{1}$, XIAOWU TAN $^{2}$ and XIUFENG ZHANG ${ }^{2}$ \\ ${ }^{1}$ Department of Respiratory Medicine, Hunan Institute of Gerontology, Hunan Province Geriatric Hospital, Changsha, \\ Hunan 410016; ${ }^{2}$ Department of Respiratory Medicine, The Second Affiliated Hospital of University of South China, \\ Hengyang, Hunan 421001, P.R. China
}

Received March 8, 2015; Accepted January 12, 2016

DOI: $10.3892 / \mathrm{mmr} .2016 .4969$

\begin{abstract}
Hypoxic pulmonary vascular remodeling (HPSR) has an important role in the development of hypoxic pulmonary hypertension. HPSR is predominantly mediated by the proliferation of pulmonary artery smooth muscle cells (PASMCs). Our previous study demonstrated that hypoxia-inducible factor (HIF)-1 $\alpha$ was able to promote the proliferation of PASMCs. Small ubiquitin-like modifier (SUMO)ylation is a post-translational modification that is important in various cellular processes. It has previously been demonstrated that HIF-1 $\alpha$ may be SUMOylated by SUMO. Conversely, SUMO-specific protease 1 (SENP-1) was able to increase the stability of HIF-1 $\alpha$ by decreasing SUMOylation of HIF-1 $\alpha$. In order to investigate whether SUMOylation of HIF-1 $\alpha$ has a role in the proliferation of PASMCs, the present study cultured PASMCs in hypoxic and normoxic chambers in vitro. The proliferation ability of PASMCs was measured using the Cell Counting kit-8 and 5-ethynyl-2'-deoxyuridine cell proliferation assays. In addition, short hairpin RNA lentiviral particles were used to knockdown the expression of SENP-1, and the expression levels of HIF-1 $\alpha$, SENP-1 and vascular endothelial growth factor (VEGF) were detected at the mRNA and protein levels using semi-quantitative polymerase chain reaction and western blotting, respectively. The present study demonstrated that SENP-1 was able to enhance the proliferative ability of PASMCs by initiating deSUMOylation of HIF-1 $\alpha$ and increasing the expression of its downstream responsive gene, VEGF.
\end{abstract}

Correspondence to: Miss. Fang Zhou or Dr Aiguo Dai, Department of Respiratory Medicine, Hunan Institute of Gerontology, Hunan Province Geriatric Hospital, 89 Guhan Road, Changsha, Hunan 410016, P.R. China

E-mail: fangzh2007@126.com

E-mail: daiaiguo2003@163.com

Key words: hypoxia-inducible factor-1 $1 \alpha$, small ubiquitin-like modifier-specific protease 1, pulmonary artery smooth muscle cells, hypoxic pulmonary hypertension

\section{Introduction}

Chronic pulmonary heart disease is a serious disease that poses a risk to human life and health, which is associated with the pathology of chronic obstructive pulmonary disease (COPD) (1). Hypoxic pulmonary hypertension (HPH) is associated with various types of lung/heart diseases, and is also prevalent in COPD (2). Pulmonary vascular proliferation and remodeling are considered to be central to the pathogenesis of HPH, during which, the proliferation of pulmonary artery smooth muscle cells (PASMCs) has an important role (3).

Previous studies have demonstrated that following exposure to low oxygen levels (hypoxia), PASMCs exhibit enhanced proliferative ability, as compared with cells exposed to normal oxygen levels (normoxia) (4-6). In our previous studies $(7,8)$, hypoxia-inducible factor (HIF)-1 $\alpha$ was shown to transcriptionally activate vascular endothelial growth factor (VEGF) in PASMCs under hypoxic conditions. HIF-1 $\alpha$, which is a subunit of the transcription factor hypoxia-inducible factor-1 (a heterodimer comprised of an alpha and a beta subunit), functions as a master regulator of the cellular and systemic homeostatic response to hypoxia via activation of the transcription of several genes, including those involved in energy metabolism, angiogenesis and apoptosis $(9,10)$.

Small ubiquitin-like modifier (SUMO) is a post-translational modification system, which functions in a manner similar to ubiquitin and regulates various cellular processes, including nuclear transport, transcriptional regulation, apoptosis and protein stability (11). In addition, SUMOylation has an important role in the regulation of HIF-1 $\alpha$ under hypoxic conditions $(12,13)$. Conversely, SUMO can be removed from proteins by SUMO-specific protease (SENP) $(14,15)$, this process is known as protein deSUMOylation. SENP-1, which is a member of the SENP family, has been reported as essential for the stability and activity of HIF-1 $\alpha$ (16). Furthermore, SENP-1 levels have been shown to increase in response to oxygen deprivation (14). Consequently, the present study aimed to investigate whether SENP-1 was able to regulate HIF-1 $\alpha$ via deSUMOylation, thus resulting in hypoxia-induced proliferation of PASMCs. 
Table I. Primers used to amplify rat SENP-1, HIF-1 $\alpha$, VEGF and $\beta$-actin mRNA, and the associated polymerase chain reaction procedures.

\begin{tabular}{|c|c|c|c|c|}
\hline $\begin{array}{l}\text { Target } \\
\text { gene }\end{array}$ & Oligonucleotide primers (5'-3') & $\begin{array}{c}\text { Annealing } \\
\text { temperature }\left({ }^{\circ} \mathrm{C}\right)\end{array}$ & Cycles & $\begin{array}{l}\text { Product } \\
\text { size (bp) }\end{array}$ \\
\hline HIF-1 $\alpha$ & $\begin{array}{l}\text { Forward TGTGGATAGCGATATGGTCAA } \\
\text { Reverse CTCTTTCCTGCTCTGTCTGGT }\end{array}$ & 53.1 & 31 & 218 \\
\hline SENP-1 & $\begin{array}{l}\text { Forward TGCAGTGCTTGATTCCGTAG } \\
\text { Reverse TTCTTTGTCCAGCGTTTCAC }\end{array}$ & 63.1 & 32 & 405 \\
\hline VEGF & $\begin{array}{l}\text { Forward CATCCACCATGCACTTGCTGT } \\
\text { Reverse GGCTGCTCCAAACTCCTTCCA }\end{array}$ & 55.8 & 28 & 178 \\
\hline$\beta$-actin & $\begin{array}{l}\text { Forward AGCCATGTACGTAGCCATCC } \\
\text { Reverse CTCTCAGCTGTGGTGGTGAA }\end{array}$ & 65 & 28 & 228 \\
\hline
\end{tabular}

HIF-1 $\alpha$, hypoxia-inducible factor-1 $\alpha$; SENP-1, small ubiquitin-like modifier-specific protease 1; VEGF, vascular endothelial growth factor.

Table II. Sequence of SENP-1 shRNA.

SENP-1 shRNA

shRNA sequence (5'-3')

SENP-1/GV118-RNAi-LV\#1

SENP-1/GV118-RNAi-LV\#2

SENP-1/GV118-RNAi-LV\#3

SENP-1/GV118-RNAi-LV\#4
GACCTCAAGTGGATTGTCAAACTCGAGTTTGACAATCCACTTGAGGTC GACCATCACACGCAAAGACATCTCGAGATGTCTTTGCGTGTGATGGTC AACCTTTGGTCAAAGTGCAAACTCGAGTTTGCACTTTGACCAAAGGTT AACCATCTAAACTGGCTCAATCTCGAGATTGAGCCAGTTTAGATGGTT

SENP-1, small ubiquitin-like modifier-specific protease 1; shRNA, short hairpin RNA.

\section{Materials and methods}

Isolation and culture of rat PASMCs. Primary PASMCs were isolated and cultured from rat intrapulmonary arteries, according to previous methods (17). Briefly, two adult male Sprague-Dawley rats (weight, 150-180 g), obtained from the Shanghai Laboratory Animal Center (Shanghai, China), were maintained under a 12-h light/dark cycle at $25^{\circ} \mathrm{C}$ and $45 \%$ humidity, with ad libitum access to food and water. The animal protocols of the present study were approved by the Ethical Committee of the Hunan Province Geriatric Hospital (Changsha, China).

The rats were sacrificed by ethyl carbamate overdose (1 $\mathrm{g} / \mathrm{kg}$; intraperitoneal injection). Distal intrapulmonary arteries were carefully dissected from the lungs of the rats in a biological safety cabinet. Adventitia and endothelium were removed from the isolated pulmonary arteries using a scalpel blade, after which the remaining smooth muscle was cut into sections $\left(<1 \mathrm{~mm}^{2}\right)$, which were further digested with $2.0 \mathrm{mg} / \mathrm{ml}$ collagenase, $0.5 \mathrm{mg} / \mathrm{ml}$ elastase (both Sigma-Aldrich, St. Louis, MO, USA) and $1.0 \mathrm{mg} / \mathrm{ml}$ bovine serum albumin (Dingguo Changsheng Biotechnology, Co., Ltd., Beijing, China) at $37^{\circ} \mathrm{C}$ in order to produce a single cell suspension of PASMCs. Subsequently, the PASMCs were cultured for 3-4 days in smooth muscle growth media [Dulbecco's modified Eagle's medium (DMEM)/F12; Gibco; Thermo Fisher Scientific, Inc., Waltham, MA, USA], supplemented with $15 \%$ fetal bovine serum (FBS; Gibco), $1 \%$ streptomycin and 1\% penicillin (Dingguo Changsheng Biotechnology, Co., Ltd.). PASMCs were cultured in a hypoxic chamber $\left(1 \% \mathrm{O}_{2}\right)$ or a normoxic chamber $(21 \% \mathrm{O} 2)$ for $2,6,12,24$ or $48 \mathrm{~h}$. The cellular purity of the culture was evaluated by observing the morphological appearance under a phase-contrast microscope, and the cells underwent immunofluorescence staining with mouse anti- $\alpha$-smooth muscle actin monoclonal antibody (AA132; Beyotime Institute of Biotechnology, Haimen, China), which was observed under a fluorescence microscope (IX73-A22FL/PH; Olympus Corporation, Tokyo, Japan). The cells used for subsequent experiments were obtained from passages $3-5$.

RNA extraction and semi-quantitative polymerase chain reaction $(P C R)$. Total RNA was isolated from the cells using TRIzol ${ }^{\circledR}$ reagent (Invitrogen; Thermo Fisher Scientific, Inc.). cDNA was synthesized from the total RNA using a Reverse Transcription system (Thermo Fisher Scientific, Inc., Pittsburgh, PA, USA), according to the manufacturer's protocol. Briefly, $1 \mu \mathrm{g}$ RNA was mixed with $1 \mu \mathrm{l}$ Oligo (dT)18 primer and nuclease-free water to a total volume of $12 \mu 1$, then centrifuged at $5000 \mathrm{x} \mathrm{g}$ for $30 \mathrm{sec}$ at $4^{\circ} \mathrm{C}$, prior to incubation at $65^{\circ} \mathrm{C}$ for $5 \mathrm{~min}$ and chilling on ice. The following components were then added to the reaction mixture to a final volume of $20 \mu \mathrm{l}$ : $4 \mu \mathrm{l}$ 5X Reaction Buffer, $1 \mu \mathrm{l}$ RiboLock RNase Inhibitor, $2 \mu 110 \mathrm{mM}$ dNTP Mix and $1 \mu \mathrm{l}$ RevertAid 
A

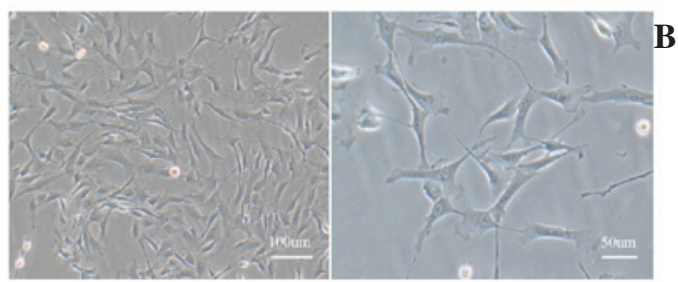

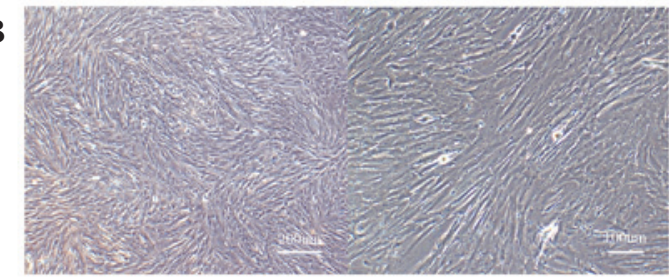

DAPI

Merge

C
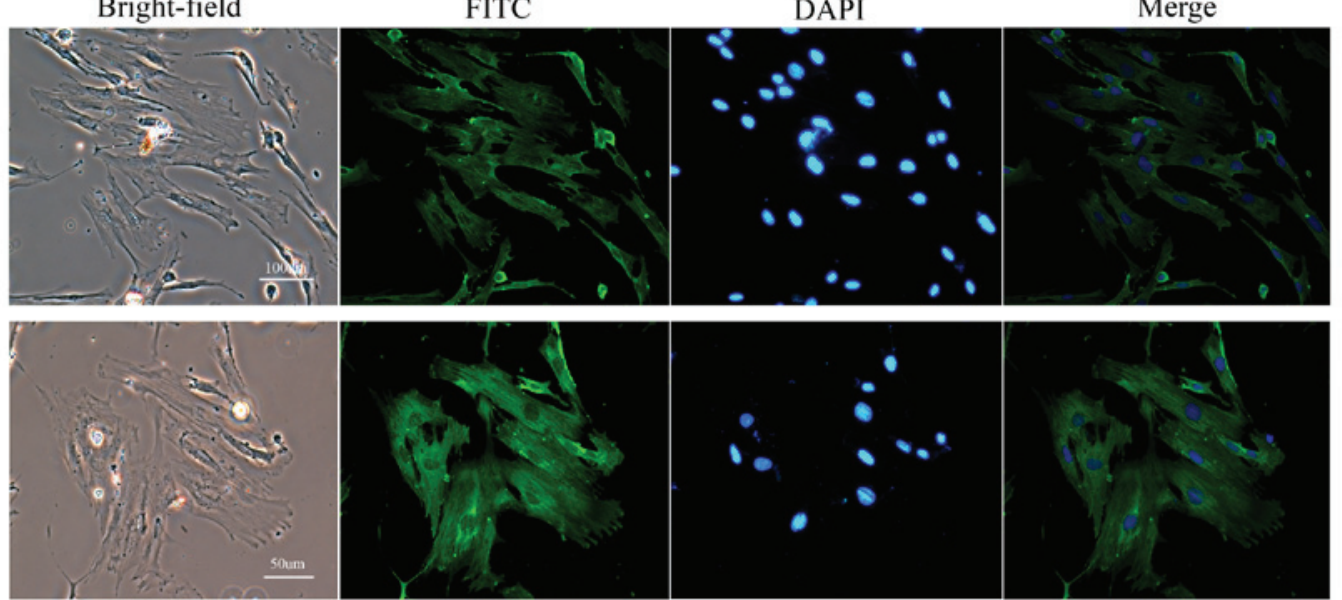

Figure 1. Morphology and identification of PASMCs. (A) Isolated cells exhibited numerous shapes, a large cytoplasm and oval-like nuclei at low confluence (magnification: left, 100x; right, 200x). (B) These cells grew in a typical 'hill-and-valley' pattern at high confluence (>70\%; magnification: left, 40x; right, 100x). (C) Immunofluorescence staining of $\alpha$-smooth muscle actin revealed that $>95 \%$ of cells were positively stained (magnification: top, 100x; bottom, 200x) PASMCs, pulmonary artery smooth muscle cells; FITC, fluorescein isothiocyanate; DAPI, 4',6-diamidino-2-phenylindole.

M-MuLV Reverse Transcriptase. The reaction mixture was incubated for $60 \mathrm{~min}$ at $42^{\circ} \mathrm{C}$, after which the reaction was terminated by incubation at $70^{\circ} \mathrm{C}$ for $5 \mathrm{~min}$. Subsequently, $2 \mu \mathrm{l}$ cDNA was amplified by PCR in a reaction mixture containing $2 \mu \mathrm{l}$ cDNA, $2 \mu 1$ each of sense and antisense primers, $25 \mu \mathrm{l}$ 2X Es Tap Master Mix (CWBio, Beijing, China) and $19 \mu \mathrm{l}$ RNase-Free Water (Dingguo Changsheng Biotechnology Co., Ltd.,), in a final volume of $50 \mu 1$. PCR was performed using the Mastercycler pro S (Eppendorf, Hamburg, Germany) and the following cycling conditions: Initialization step $\left(94^{\circ} \mathrm{C}\right.$ for $2 \mathrm{~min})$; denaturation step $\left(94^{\circ} \mathrm{C}\right.$ for $\left.30 \mathrm{sec}\right)$; annealing step (30 sec); elongation step $\left(72^{\circ} \mathrm{C}\right.$ for $\left.30 \mathrm{sec}\right)$; and final elongation step $\left(72^{\circ} \mathrm{C}\right.$ for $\left.2 \mathrm{~min}\right)$. $\beta$-actin was amplified in parallel as an internal control. The primers were purchased from Sangon Biotech Co., Ltd. (Shanghai, China). The primer sequences, annealing temperatures and cycle numbers are presented in Table I. The PCR products were separated by $1 \%$ agarose gel electrophoresis (100 V; $30 \mathrm{~min})$ and images of the gels were captured using the ChemiDoc XRS+ system (Bio-Rad Laboratories, Inc., Hercules, CA, USA). Densitometric analyses were performed using ImageJ 2x software (National Institutes of Health, Bethesda, MD, USA).

Western blot analysis. Total protein was extracted from PASMCs using radioimmunoprecipitation assay lysis buffer supplemented with phosphatase inhibitors (Roche Diagnostics $\mathrm{GmbH}$, Mannheim, Germany). Nucleoprotein was obtained using the Nucleoprotein Protein Extraction kit (Beyotime Institute of Biotechnology). The protein concentration of the cell lysates was quantified using bicinchoninic acid protein assay (Beyotime Institute of Biotechnology). Approximately

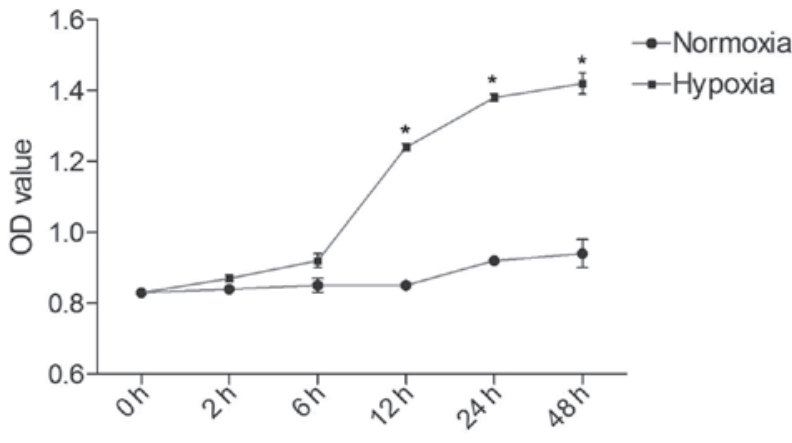

Figure 2. Proliferative ability of PASMCs in the hypoxic and normoxic groups was detected by Cell Couunting kit- 8 assay. PASMCs in the hypoxic group exhibited a faster growth speed, as compared with the normoxic group, particularly after $6 \mathrm{~h}$. Data are presented as the mean \pm standard deviation. ${ }^{*}<<0.05$. PASMCs, pulmonary artery smooth muscle cells; OD, optical density.

$40 \mu \mathrm{g}$ protein was separated by $8 \%$ sodium dodecyl sulfate-polyacrylamide gel electrophoresis and was transferred to polyvinylidene difluoride membranes (EMD Millipore, Billerica, MA, USA). The membranes were blocked in $5 \%$ nonfat milk with $0.2 \%$ Tween-20, and were then incubated with the following primary antibodies: Anti- $\alpha$-smooth muscle actin ( $\alpha$-SMA; 1:500), anti-histone 3 (1:500; AH433; Beyotime Institute of Biotechnology); anti-SENP-1 (1:300; sc-67074), anti-VEGF (1:500; sc-507; Santa Cruz Biotechnology, Inc., Dallas, TX, USA); anti- $\beta$-actin (1:500; BM0627; Wuhan Boster Biological Technology, Ltd., Wuhan, China); and anti-HIF-1 $\alpha$ (1:1,000; NB100-479; Novus International, St Charles, MO, USA). The membranes were then blotted with 
A

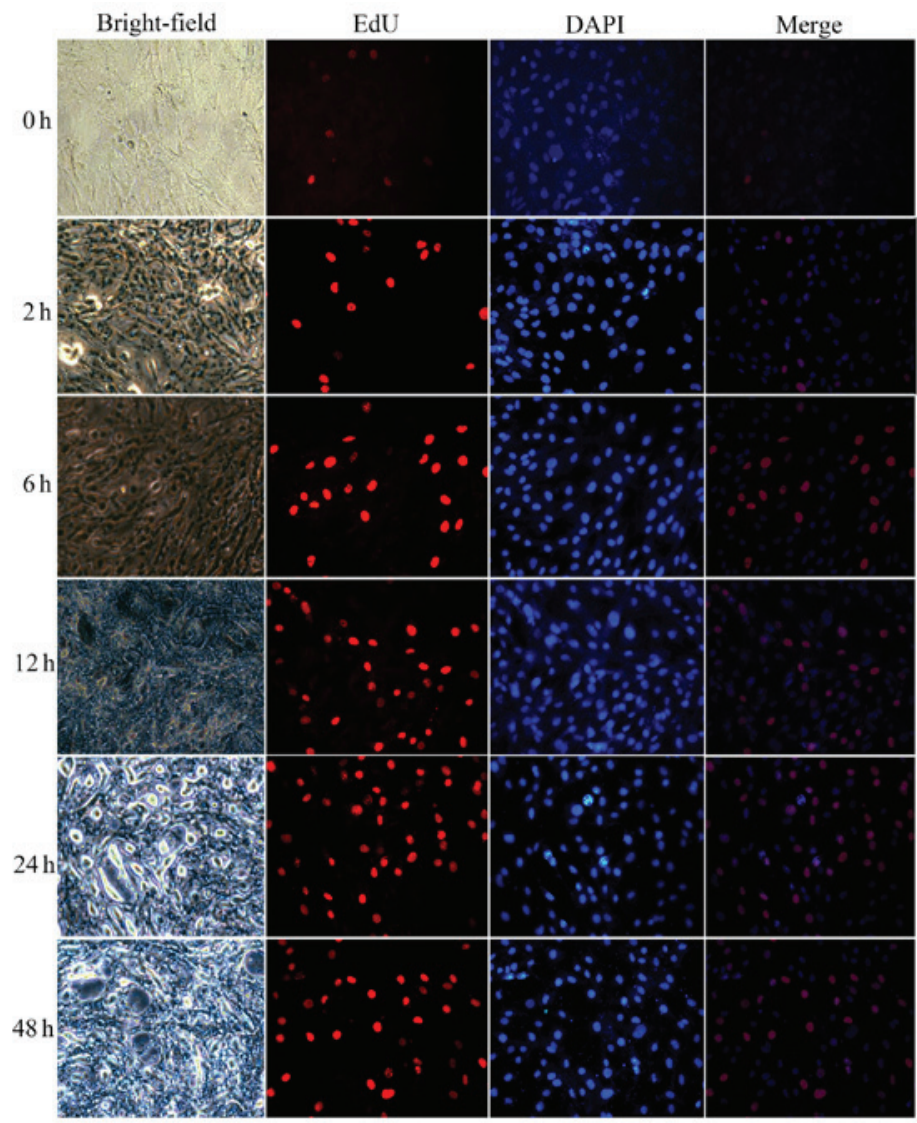

B

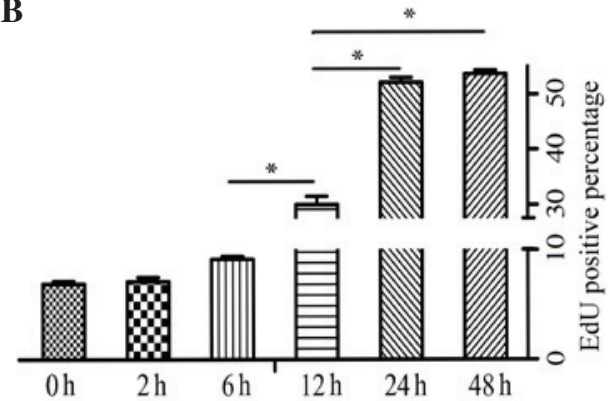

Figure 3. (A) Proliferative ability of pulmonary artery smooth muscle cells in the hypoxic and normoxic groups, as detected by EdU assay (magnification, $\mathrm{x} 100$ ). The number of EdU-positive cells increased as hypoxic exposure time was prolonged. (B) The positive rates were as follows: Normoxia, $6.86 \pm 0.25 \%$; hypoxia 2 h, $7.08 \pm 0.38 \%$; hypoxia 6 h, $9.11 \pm 0.24 \%$; hypoxia 12 h, 29.99 $\pm 1.48 \%$; hypoxia 24 h, $52.12 \pm 0.89 \%$; hypoxia 48 h, $53.69 \pm 0.64 \%$. Data are expressed as the mean \pm standard deviation, $\mathrm{n}=5$. * $\mathrm{P}<0.05$. EdU, 5-ethynyl-2'-deoxyuridine; DAPI, 4',6-diamidino-2-phenylindole.

horseradish peroxidase-conjugated goat anti-rabbit $(1: 4,000$; sc-2004) and goat anti-mouse (1:4,000; sc-2005) immunoglobulin G secondary antibodies (Santa Cruz Biotechnology, Inc.). Subsequently, the membranes were extensively washed with phosphate-buffered saline (PBS) containing $0.1 \%$ Tween and were exposed to an enhanced chemiluminescent reagent (Beyotime Institute of Biotechnology). After scanning the X-ray film, the optical density of the immunoblots was calculated using ImageJ 2x software. Histone 3 was employed as the loading control for the nucleoprotein and $\beta$-actin was used as the loading control for total protein.

Immunofluorescence assay. PASMCs were fixed in medium containing $4 \%$ paraformaldehyde for $30 \mathrm{~min}$. The cells were permeabilized using $0.2 \%$ Triton $\mathrm{X}-100$ for $2 \mathrm{~min}$, and were blocked with $5 \%$ bovine serum albumin (Dingguo Changsheng Biotechnology,Co.,Ltd.)for $30 \mathrm{~min}$. The cells were then incubated with $\alpha$-SMA antibody (Beyotime Institute of Biotechnology) at $4^{\circ} \mathrm{C}$ overnight, were washed with PBS, and were incubated with streptavidin biotin complex-fluorescein isothiocyanate (Wuhan Boster Biological Technology, Ltd.) for $30 \mathrm{~min}$ at $37^{\circ} \mathrm{C}$. After counterstaining with 4',6-diamidino-2-phenylindole (DAPI), immunofluorescence was observed and images were captured under a fluorescence microscope (IX73-A22FL/PH).

Cell Counting kit (CCK)-8 assay. A $100 \mu 1$ PASMCs suspension $\left(1 \times 10^{4}\right.$ cells/well) was seeded into 96 -well plates. Once confluence reached $70 \%$, the cells were synchronized in DMEM/F12 without FBS for $24 \mathrm{~h}$. Subsequently, the medium was replaced with DMEM containing FBS and the plates were incubated in a hypoxic or normoxic chamber. The plates were incubated for an appropriate duration $(2,6,12$, 24 or $48 \mathrm{~h}$ ) in the incubator (5 duplicate wells for each time point). Subsequently, $10 \mu \mathrm{l}$ CCK-8 solution (CCK-8 Assay kit; Wuhan Boster Biological Technology, Ltd.) was added to each well and the plates were incubated for $4 \mathrm{~h}$ at $37^{\circ} \mathrm{C}$. The absorbance was then measured at a wavelength of $460 \mathrm{~nm}$ using a microplate autoreader (EL309; BioTek Instruments, Inc., Winooski, VT, USA). All of the experiments were repeated at least 3 times.

5-ethynyl-2'-deoxyuridine (EdU) cell proliferation assay. PASMCs were seeded into 96 well plates $\left(1 \times 10^{4}\right.$ cells/well). Following synchronization in DMEM/F12 without FBS for $24 \mathrm{~h}$, the cells were separately incubated in a normoxic or hypoxic chamber for an appropriate length of time (2, 6, 12, 24 or $48 \mathrm{~h}$ ). Subsequently, $100 \mu \mathrm{l}$ EdU (50 $\mu \mathrm{M}$; Guangzhou Ribobio Co., Ltd., Guangzhou, China) was added to the wells and the plates were incubated in the appropriate chamber for $2 \mathrm{~h}$, according to the manufacturer's protocol. After EdU staining, the cells were counterstained with DAPI. EdU-positive cells were imaged randomly by fluorescence microscopy, and the EdU-positive cells were randomly counted in 5 fields. The ratio of positive cells was calculated. 


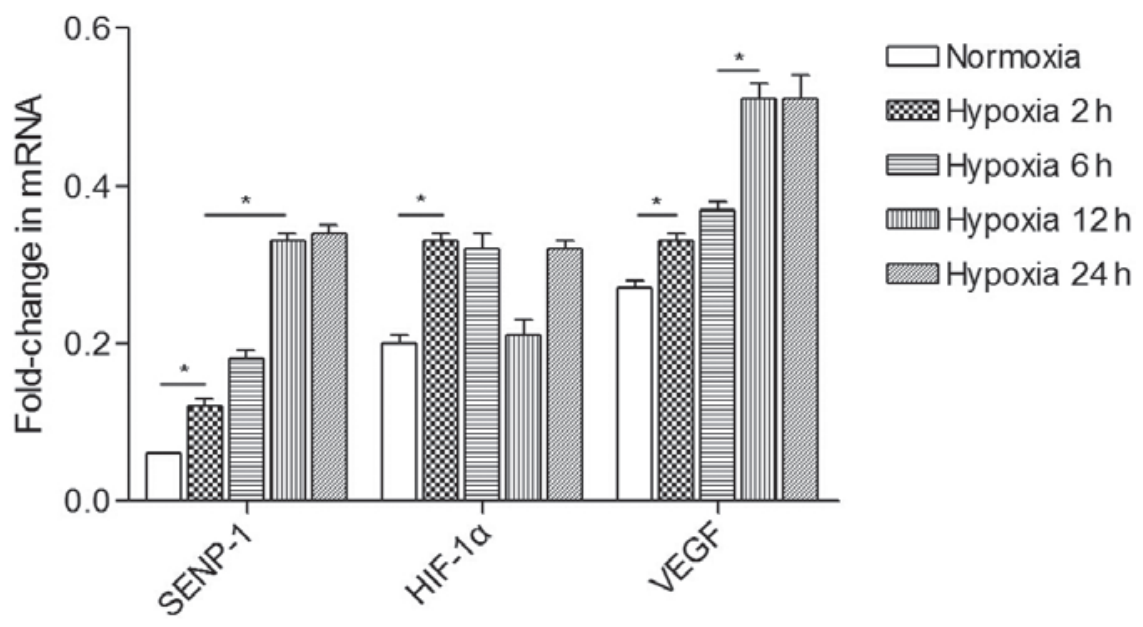

Figure 4. mRNA expression levels of SENP-1, HIF-1 $\alpha$ and VEGF in pulmonary artery smooth muscle cells under normoxic and hypoxic conditions. Increasing expression levels of SENP-1, HIF-1 $\alpha$ and VEGF were detected in the hypoxic group as exposure time was prolonged. Data are presented as the mean \pm standard deviation. ${ }^{*} \mathrm{P}<0.05$. SENP-1, small ubiquitin-like modifier-specific protease 1; HIF-1 $\alpha$, hypoxia-inducible factor-1 $\alpha$; VEGF, vascular endothelial growth factor.

SENP-1 short hairpin (sh)RNA lentiviral synthesis. SENP-1 shRNA and scrambled shRNAs were designed and synthesized by Shanghai Genechem Co., Ltd. (Shanghai, China). The lentiviral particles were also produced by Shanghai Genechem Co., Ltd. Primary cultured PASMCs in DMEM/F12 medium were infected with lentiviral particles for $24 \mathrm{~h}$. Cells were then cultured with puromycin $(4 \mu \mathrm{M}$; Shanghai Genechem Co., Ltd.) for $48 \mathrm{~h}$, in order to kill the uninfected cells and to ensure $>95 \%$ infection of cells. Four types of shRNA were used, as follows: SENP-1/GV118-RNAi-LV\#1, SENP-1/GV118-RNAi-LV\#2, SENP-1/GV118-RNAi-LV\#3, SENP-1/GV118-RNAi-LV\#4 (Table II). The knockdown efficiency of each clone was determined using western blotting. The clones with the maximum knockdown efficiency were selected for subsequent experiments. As a control, cells were infected with lentiviral particles containing scrambled shRNAs. Uninfected cells were used as a blank group.

Statistical analysis. All data were analyzed using SPSS 10.0 software (SPSS Inc., Chicago, IL, USA). The statistical significance of the differences between the mean values was determined using one-way analysis of variance. The data were examined for equality of variance, followed by either Fisher test (if variances were equal) or approximate variance $\mathrm{F}$ test/Welch method (if variances were unequal). To compare pairwise difference, Fisher's least significant difference test was used. In the case of homogeneity of variance, Dunnett's T3 was used. Data are presented as the mean \pm standard deviation. $\mathrm{P}<0.05$ was considered to indicate a statistically significant difference.

\section{Results}

Morphology and identification of PASMCs. Following isolation and culture for 3-4 days, PASMCs dissociated from the tissue exhibited numerous shapes, an abundant cytoplasm and oval-like nuclei (Fig. 1A). After 7-10 days, PASMCs were spindle-shaped, arranged in bundles, and grew in a typical 'hill-and-valley' pattern at high confluence (Fig. 1B). To further characterize these cells, immunofluorescence was used

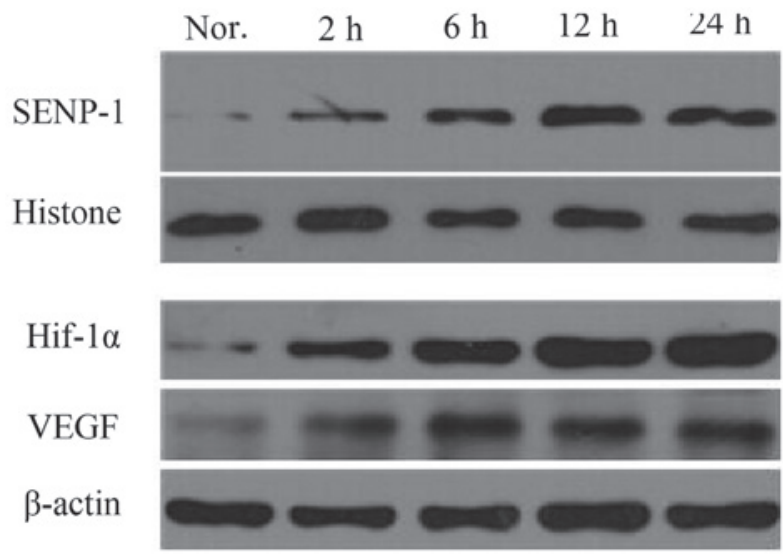

Figure 5. Protein expression levels of SENP-1, HIF-1 $\alpha$ and VEGF in pulmonary artery smooth muscle cells under normoxic and hypoxic conditions. Nor., normoxic group; SENP-1, small ubiquitin-like modifier-specific protease 1 ; HIF-1 $\alpha$, hypoxia-inducible factor-1 $\alpha$; VEGF, vascular endothelial growth factor.

to detect $\alpha$-SMA. A strong green fluorescence was detected in the cytoplasm, and $>95 \%$ of cells were positively stained (Fig. 1C). These results indicate that the separated cells were PASMCs.

Hypoxia promotes the proliferative ability of PASMCs. To determine whether hypoxia was able to influence the proliferative ability of PASMCs, a CCK-8 assay was used to measure the proliferation of cells cultured in hypoxic and normoxic conditions. As shown in Fig. 2, the optical density (OD) value of each group increased in a time-dependent manner. In the hypoxic group, the OD value at 12,24 and $48 \mathrm{~h}$ was markedly higher than at 2 and $6 \mathrm{~h}(\mathrm{P}<0.05)$. After reaching a peak at $24 \mathrm{~h}$, proliferative speed tended to slow, resulting in a flat growth curve. PASMCs cultured in normoxic conditions exhibited a slower multiplication speed, as compared with the hypoxic group. There were statistical differences between hypoxic and normoxic groups at 12,24 and $48 \mathrm{~h}(\mathrm{P}<0.05)$.

Similar results were obtained from the EdU staining assay (Fig. 3A). Under normoxic conditions, a small proportion of 
A

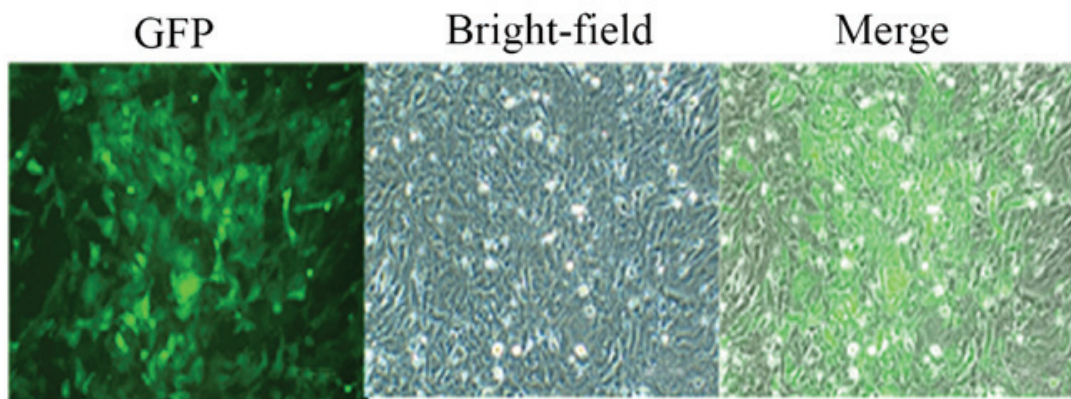

B

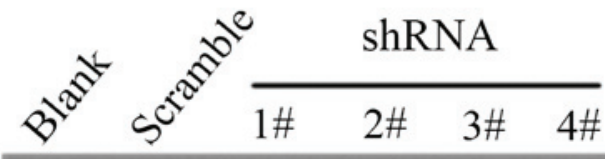

SENP-1

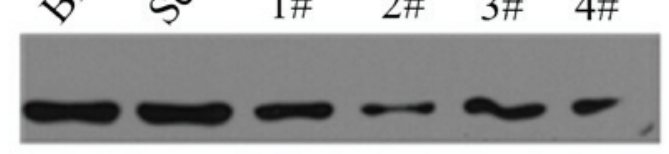

Histone

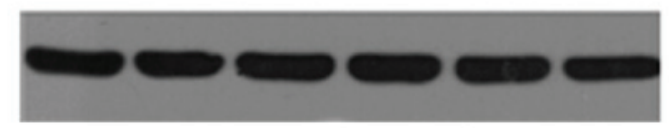

Figure 6. Pulmonary artery smooth muscle cells were infected with SENP-1 shRNA lentiviruses. (A) Cells infected with lentivirus were labeled with GFP (magnification, x100). (B) Western blotting was performed to confirm the knockdown efficiency of the shRNA lentiviruses, and SENP-1/GV118-RNAi-LV2\# exhibited the maximum reduction of SENP-1. SENP-1, small ubiquitin-like modifier-specific protease 1; GFP, green fluorescent protein; shRNA short hairpin RNA.

A

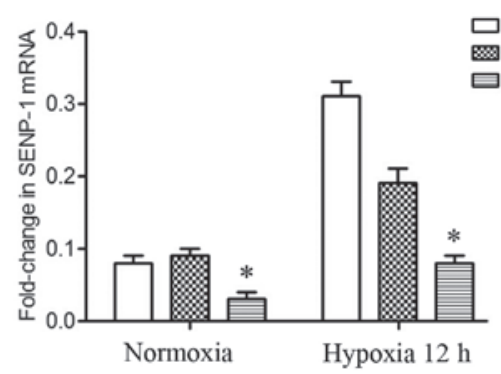

C

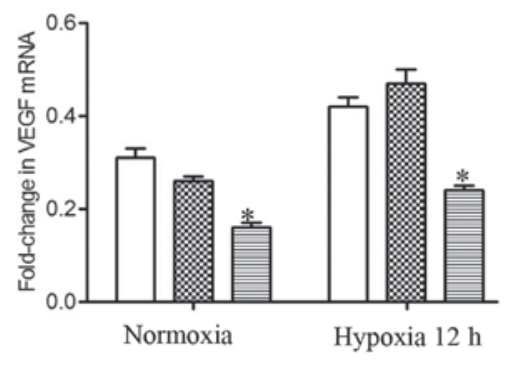

B

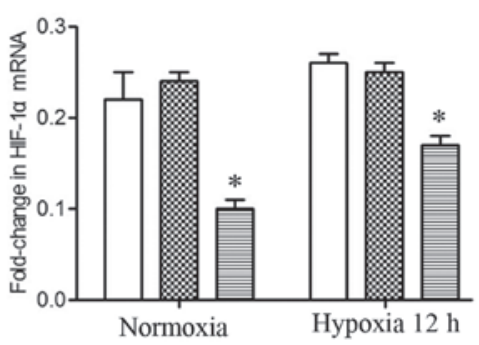

D

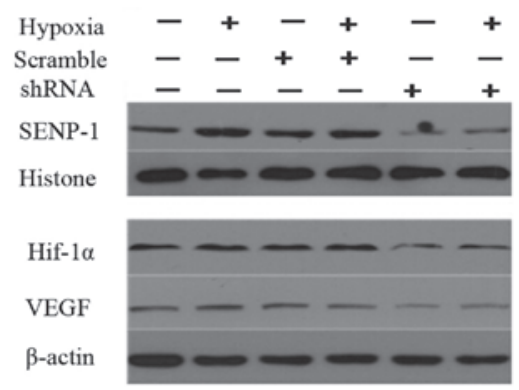

Figure 7. (A-C) mRNA and (D) protein expression levels of (A) SENP-1, (B) HIF-1 $\alpha$ and (C) VEGF in pulmonary artery smooth muscle cells with SENP-1 knockdown exposed to hypoxia for $12 \mathrm{~h}$. In the SENP-1 shRNA group, HIF-1 $\alpha$ and VEGF exhibited a downregulation at the mRNA and protein level, as compared with the scramble and blank groups under hypoxic conditions. Data are presented as the mean \pm standard deviation. ${ }^{*}<0.05$ vs. the scramble and blank groups. SENP-1, small ubiquitin-like modifier-specific protease 1; HIF-1 $\alpha$, hypoxia-inducible factor-1 $\alpha$; VEGF, vascular endothelial growth factor; shRNA, short hairpin RNA.

PASMCs were labeled with EdU. Although no obvious distinction was observed in the hypoxic group at 2 or $6 \mathrm{~h}$ compared with the normoxic group, fluorescence was markedly increased in hypoxic PASMCs at 12, 24 and $48 \mathrm{~h}$. There was a statistical difference in EdU positive staining between 24, 48 and $12 \mathrm{~h}$ $(\mathrm{P}<0.05)$, whereas no significant difference was detected between 24 and $48 \mathrm{~h}$ (Fig. 3B).
Hypoxia-induced proliferation of PASMCs is mediated by SENP-1 via the HIF-1-VEGF-dependent pathway. To investigate whether the effects on proliferation were mediated by deSUMOylation of HIF-1 $\alpha$, semi-quantitative PCR was performed to analyze the mRNA expression levels of SENP-1, HIF-1 $\alpha$ and VEGF in PASMCs (Fig. 4). The mRNA expression levels of SENP-1 at $2 \mathrm{~h}$ in the hypoxic group were increased, 


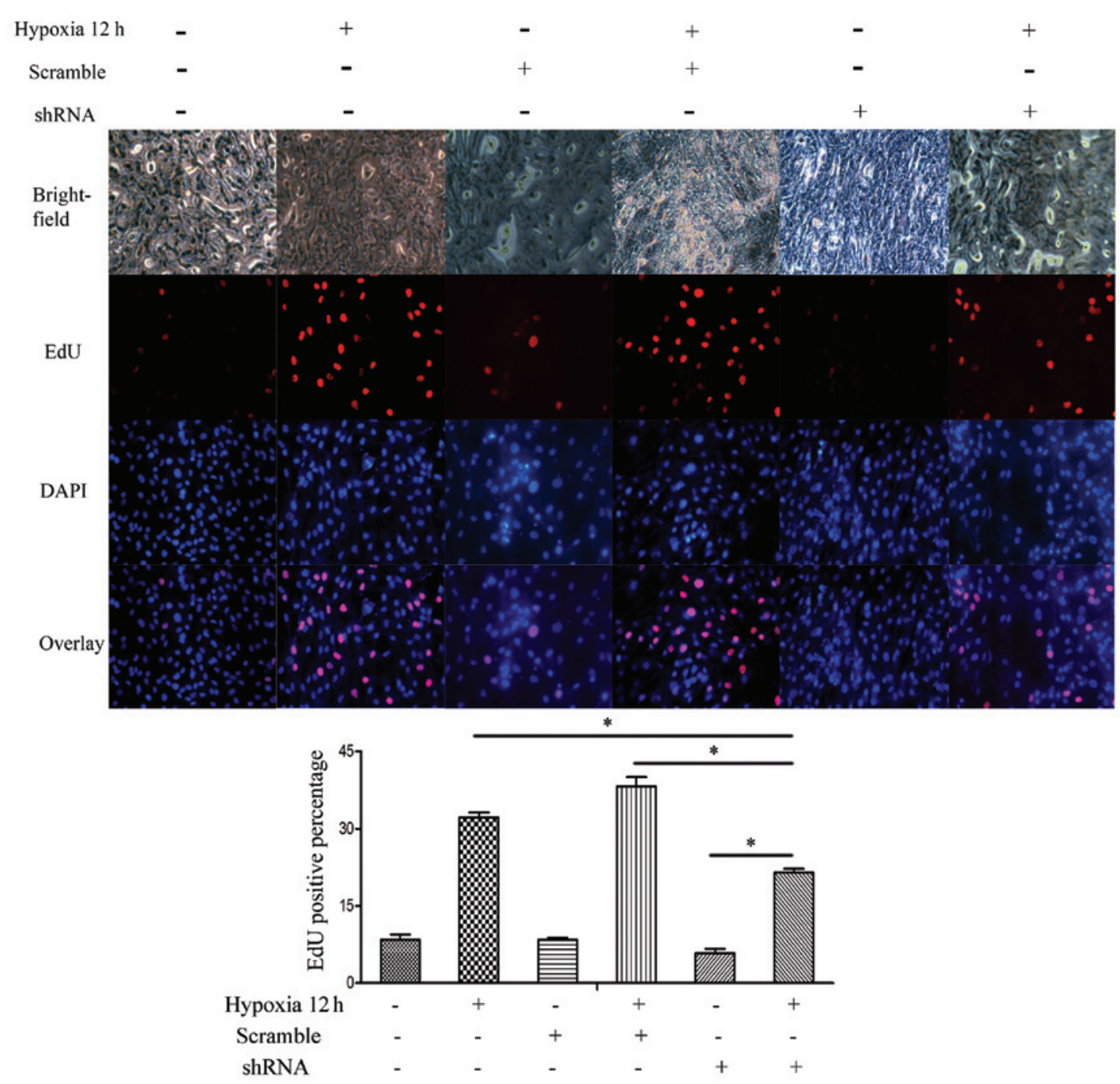

Figure 8. An EdU assay was conducted to determine the proliferation of pulmonary artery smooth muscle cells with SENP-1 knockdown exposed to hypoxia for $12 \mathrm{~h}$ (magnification, x100). In the SENP-1 shRNA group, less EdU-positive cells were detected, as compared with in the scramble and blank groups under hypoxic conditions. "P<0.05. SENP-1, small ubiquitin-like modifier-specific protease 1; EdU, 5-ethynyl-2'-deoxyuridine; DAPI, 4',6-diamidino-2-phenylindole; shRNA, short hairpin RNA.

as compared with in the normoxic group $(\mathrm{P}<0.05)$. In addition, the mRNA expression levels of SENP-1 were upregulated at 12 and $24 \mathrm{~h}$ compared with at 2 and $6 \mathrm{~h}(\mathrm{P}<0.05)$. The mRNA expression levels of HIF-1 $\alpha$ in the hypoxic group at 2, 6 and $24 \mathrm{~h}$ were increased, as compared with in the normoxic group. The mRNA expression levels of VEGF in the hypoxic group were examined at 2,6,12 and $24 \mathrm{~h}$. After PASMCs were exposed to hypoxia for $2 \mathrm{~h}$, a marked increased in VEGF mRNA expression was detected. In addition, a further increase in VEGF mRNA expression was observed as the hypoxic treatment time was prolonged.

Western blotting was used to detect protein expression levels in the cells under normoxic and hypoxic conditions (Fig. 5). Following $2 \mathrm{~h}$ of hypoxic exposure, the expression levels of SENP-1 were elevated, as compared with the normoxic group. Furthermore, SENP-1 exhibited a time-dependent increase, and the difference between 12 and $24 \mathrm{~h}$, and 2 and $6 \mathrm{~h}$ was markedly different. Similar to SENP-1, hypoxic treatment led to increased expression levels of HIF-1 $\alpha$ and VEGF. As treatment time was prolonged, the expression levels reached a peak at 12 or $24 \mathrm{~h}$, which was markedly higher, as compared with 2 or $6 \mathrm{~h}$.
To demonstrate the effects of downregulation of SENP-1 in hypoxic conditions, shRNA was used to study the effects of SENP-1 knockdown on PASMCs. Since the shRNA lentivirus was labeled with green fluorescent protein, PASMCs infected with the lentivirus could easily by identified by fluorescence microscopy (Fig. 6A). Subsequently, western blotting was conducted to confirm the efficiency of SENP-1 shRNA targeting. As shown in Fig. 6B, SENP-1/GV118-RNAi-LV\#2 exhibited the maximum reduction of SENP-1 expression, and was therefore selected for subsequent experiments.

Following knockdown of SENP-1 (Fig. 7A), PASMCs were incubated in a hypoxic chamber for $12 \mathrm{~h}$. Subsequently, total RNA was extracted from the cells and semi-quantitative PCR was performed to detect the mRNA expression levels of HIF-1 $\alpha$ and VEGF. The mRNA expression levels of HIF-1 $\alpha$ and VEGF were significantly downregulated in the SENP-1 shRNA group, as compared with the scramble and blank groups (Fig. 7B and C; $\mathrm{P}<0.05$ ). In addition, no difference was observed between the scrambled and blank groups. As presented in Fig. 7D, the protein expression levels of HIF-1 $\alpha$ and VEGF were also downregulated in the SENP-1 shRNA 
group, as compared with in the scrambled and blank groups. These results indicate that knockdown of SENP-1 leads to a marked decrease in the mRNA and protein expression levels of HIF-1 $\alpha$ and VEGF.

To further evaluate the effects of SENP-1 knockdown on PASMCs, an EdU assay was conducted to compare the proliferative ability among the SENP-1 shRNA, scrambled and blank groups. As shown in Fig. 8, compared with the scrambled and blank groups, there was a declining ratio of EdU-positive cells in the SENP-1 shRNA group $(\mathrm{P}<0.05)$. Furthermore, the number of EdU-positive cells in the SENP-1 shRNA group was significantly higher at $12 \mathrm{~h}$ (hypoxia) than at $0 \mathrm{~h}$ (normoxia) $(\mathrm{P}<0.05)$. Consequently, these results suggest that knockdown of SENP-1 may exhibit an inhibitory effect on the proliferation of PASMCs in a hypoxic environment.

\section{Discussion}

HIF-1 is a master regulator of oxygen homeostasis, which is associated with numerous biological processes, starting in early embryonic development and extending into adult life (18). In response to hypoxia, HIF-1 $\alpha$, a subunit of HIF-1, dimerizes with HIF-1 $\beta$, resulting in the formation of activated HIF-1 protein, which can bind to enhancer sequences in target genes that contain the core RCGTG binding motif $(19,20)$. VEGF is a critical downstream target gene of HIF-1, which is essential for HIF-1-mediated neovascularization $(21,22)$. Therefore, the HIF-1-VEGF-dependent pathway is considered to have an important role in the proliferation of PASMCs.

Protein SUMOylation is regarded as an important mechanism of post-translational modification (12). Hypoxia is able to induce the SUMOylation of HIF-1 $\alpha$, thus mediating its activity and stability (13). However, the role of SUMOylation in the regulation of HIF- $1 \alpha$ stability remains controversial (12). Bae et al (23) demonstrated that the protein level and transcriptional activity of HIF-1 $\alpha$ were upregulated by SUMO-1. In addition, RSUME has been reported to participate in HIF-1 $\alpha$ stabilization by enhancing HIF-1 $\alpha$ SUMOylation (24). However, a previous study indicated that SUMO modification of HIF-1 $\alpha$ was able to repress its transcriptional activity (25). In addition, SUMOylation of HIF-1 $\alpha$, which binds to von Hippel-Lindau protein in a hydroxyl proline-independent manner, has been shown to lead to ubiquitination and proteasomal degradation of HIF-1 $\alpha$. The opposite process of SUMOylation, namely deSUMOylation, has also been suggested to exert huge influence in stabilizing and activating HIF-1 $\alpha(26,27)$. Consequently, it is of great significance to determine how HIF- $1 \alpha$ is post-transcriptionally modified by SENP-1 in PASMCs under hypoxic conditions.

The present study demonstrated that upon exposure to hypoxia, the proliferation of PASMCs increased in a time-dependent manner. Simultaneously, the mRNA and protein expression levels of SENP-1, HIF-1 $\alpha$ and VEGF were upregulated. Following knockdown of SENP-1, hypoxia-induced proliferation of PASMCs was markedly inhibited, and HIF-1 $\alpha$ and VEGF expression was decreased at both the mRNA and protein level. In conclusion, hypoxia may significantly promote the proliferation of rat PASMCs, which is regulated by SENP-1, via the HIF-1-VEGF-dependent pathway. Therefore, targeting SENP-1-mediated deSUMOylation may be considered an effective method for the suppression of the pathogenesis of HPH.

\section{Acknowledgements}

The authors acknowledge the work of all investigators involved in the present study. This study was supported by a grant from the National Natural Science Foundation of China (grant no. 30971329).

\section{References}

1. Vestbo J,Hurd SS, Agusti AG, Jones PW, Vogelmeier C, Anzueto A Barnes PJ, Fabbri LM, Martinez FJ, Nishimura M, et al: Global strategy for the diagnosis, management, and prevention of chronic obstructive pulmonary disease: GOLD executive summary. Am J Respir Crit Care Med 187: 347-365, 2013.

2. Wright JL, Levy RD and Churg A: Pulmonary hypertension in chronic obstructive pulmonary disease: Current theories of pathogenesis and their implications for treatment. Thorax 60: 605-609, 2005.

3. Jin H, Wang Y, Zhou L, Liu L, Zhang P, Deng W and Yuan Y: Melatonin attenuates hypoxic pulmonary hypertension by inhibiting the inflammation and the proliferation of pulmonary arterial smooth muscle cells. J Pineal Res 57: 442-450, 2014.

4. Sarkar J, Gou D, Turaka P, Viktorova E, Ramchandran R and Raj JU: MicroRNA-21 plays a role in hypoxia-mediated pulmonary artery smooth muscle cell proliferation and migration. Am J Physiol Lung Cell Mol Physiol 299: L861-L871, 2010.

5. Chen B, Calvert AE, Cui H and Nelin LD: Hypoxia promotes human pulmonary artery smooth muscle cell proliferation through induction of arginase. Am J Physiol Lung Cell Mol Physiol 297: L1151-L1159, 2009.

6. Growcott EJ, Banner KH and Wharton J: Hypoxia amplifies the proliferative capacity of distal human pulmonary artery smooth-muscle cells. Chest 128 (6 Suppl): 600S-601S, 2005.

7. Li QF and Dai AG: Hypoxia-inducible factor-1 alpha regulates the role of vascular endothelial growth factor on pulmonary arteries of rats with hypoxia-induced pulmonary hypertension. Chin Med J (Engl) 117: 1023-1028, 2004.

8. Li QF and Dai AG: Hypoxia inducible factor-1 alpha correlates the expression of heme oxygenase 1 gene in pulmonary arteries of rat with hypoxia-induced pulmonary hypertension. Acta Biochim Biophys Sin (Shanghai) 36: 133-140, 2004.

9. Zhang R, Wu Y, Zhao M, Liu C, Zhou L, Shen S, Liao S Yang K, Li Q and Wan H: Role of HIF-1alpha in the regulation ACE and ACE2 expression in hypoxic human pulmonary artery smooth muscle cells. Am J Physiol Lung Cell Mol Physiol 297: L631-L640, 2009.

10. Rey S and Semenza GL: Hypoxia-inducible factor-1-dependent mechanisms of vascularization and vascular remodelling. Cardiovasc Res 86: 236-242, 2010.

11. Saitoh H and Hinchey J: Functional heterogeneity of small ubiquitin-related protein modifiers SUMO-1 versus SUMO-2/3. J Biol Chem 275: 6252-6258, 2000.

12. Liu B and Shuai K: Regulation of the sumoylation system in gene expression. Curr Opin Cell Biol 20: 288-293, 2008.

13. Yee Koh M, Spivak-Kroizman TR and Powis G: HIF-1 regulation: Not so easy come, easy go. Trends Biochem Sci 33: 526-534, 2008.

14. Xu Y, Zuo Y, Zhang H, Kang X, Yue F, Yi Z, Liu M, Yeh ET, Chen $\mathrm{G}$ and Cheng J: Induction of SENP1 in endothelial cells contributes to hypoxia-driven VEGF expression and angiogenesis. J Biol Chem 285: 36682-36688, 2010.

15. Hickey CM, Wilson NR and Hochstrasser M: Function and regulation of SUMO proteases. Nat Rev Mol Cell Biol 13: 755-766, 2012.

16. Gu J, Fan Y, Liu X, Zhou L, Cheng J, Cai R and Xue S: SENP1 protects against myocardial ischaemia/reperfusion injury via a HIF1 $\alpha$-dependent pathway. Cardiovasc Res 104: 83-92, 2014. 
17. Wang J, Shimoda LA and Sylvester JT: Capacitative calcium entry and TRPC channel proteins are expressed in rat distal pulmonary arterial smooth muscle. Am J Physiol Lung Cell Mol Physiol 286: L848-L858, 2004.

18. Semenza GL: Hypoxia-inducible factor 1: Control of oxygen homeostasis in health and disease. Pediatr Res 49: 614-617, 2001.

19. Ortiz-Barahona A, Villar D, Pescador N, Amigo J and del Peso L: Genome-wide identification of hypoxia-inducible factor binding sites and target genes by a probabilistic model integrating transcription-profiling data and in silico binding site prediction. Nucleic Acids Res 38: 2332-2345, 2010.

20. Mole DR, Blancher C, Copley RR, Pollard PJ, Gleadle JM, Ragoussis $\mathbf{J}$ and Ratcliffe PJ: Genome-wide association of hypoxia-inducible factor (HIF)-1alpha and HIF-2alpha DNA binding with expression profiling of hypoxia-inducible transcripts. J Biol Chem 284: 16767-16775, 2009.

21. Oladipupo S, Hu S, Kovalski J, Yao J, Santeford A, Sohn RE, Shohet R, Maslov K, Wang LV and Arbeit JM: VEGF is essential for hypoxia-inducible factor-mediated neovascularization but dispensable for endothelial sprouting. Proc Nat Acad Sci USA 108: 13264-13269, 2011.
22. Cucina A, Borrelli V, Randone B, Coluccia P, Sapienza P and Cavallaro A: Vascular endothelial growth factor increases the migration and proliferation of smooth muscle cells through the mediation of growth factors released by endothelial cells. J Surg Res 109: 16-23, 2003.

23. Bae SH, Jeong JW, Park JA, Kim SH, Bae MK, Choi SJ and Kim KW: Sumoylation increases HIF-1alpha stability and its transcriptional activity. Biochem Biophys Res Commun 324: 394-400, 2004.

24. Carbia-Nagashima A, Gerez J, Perez-Castro C, Paez-Pereda M, Silberstein S, Stalla GK, Holsboer F and Arzt E: RSUME, a small RWD-containing protein, enhances SUMO conjugation and stabilizes HIF-1alpha during hypoxia. Cell 131: 309-323, 2007.

25. Berta MA, Mazure N, Hattab M, Pouysségur J and Brahimi-Horn MC: SUMOylation of hypoxia-inducible factor-1alpha reduces its transcriptional activity. Biochem Biophys Res Commun 360: 646-652, 2007.

26. Cheng J, Kang X, Zhang S and Yeh ET: SUMO-specific protease 1 is essential for stabilization of HIF1alpha during hypoxia. Cell 131: 584-595, 2007.

27. Yeh ET: SUMOylation and De-SUMOylation: Wrestling with life's processes. J Biol Chem 284: 8223-8227, 2009. 